\title{
Jenseitsvorstellungen zur Lutherzeit
}

\author{
von JÖRG BREHMER
}

Die Hölle hat heute an Bedeutung und Schreckenskraft verloren. Und dennoch: Wir begegnen dem diabolischen Bodenpersonal auf Du und Du, auf Schritt und Tritt. Im Unterhaltungsfernsehen - derzeit mit einer neuen Serie „Lucifer. Hot as hell“" Staffel 1-3 - in der Werbung (,Putzteufel“) und wenn ich mich an meine eigene Schulzeit in den 1980er Jahren zurückerinnere, sogar in Lernmaterialien („Uli der Fehlerteufel“). Wir leben offenbar in einem säkularen Zeitalter - der Teufel kann uns nicht mehr schrecken, er unterhält, lässt sich vertreiben, vermeiden oder man kann ihm gar mit (Rechtschreib-)Strategien beikommen. Es ist heute ein Leichtes, dass eine 10-Jährige den (Fehler-)teufel besiegen kann.

Wenn wir aber an die Anfänge der sogenannten Neuzeit gehen - jedenfalls in eine relative Nähe, auf was sich Historiker verständigt haben - so finden wir in dieser Umbruchzeit ein anderes, uns heute fremdes Welt- und Jenseitsbild. Die Schwelle aus dem Mittelalter heraus datieren wir ungefähr vor nunmehr fünfhundert Jahren: Historische Meilensteine sind etwa die Entwicklung des Buchdrucks oder große - aus europäischer Sicht - Entdeckerfahrten wie die zum amerikanischen Kontinent oder auch die Reformation(en) - v.a. die um und mit Martin Luther. Es ist ein „Schwanken zwischen Aufbruchsstimmung und Angstgefühl"“.

Heute sehen wir Martin Luther gerne als einen von uns, als einen Medienstrategen, ${ }^{2}$ als einen, der die Neuzeit und unser Religions- bzw. Konfessions- und Weltverständnis geprägt hat. Bei genauerer Betrachtung aber müssen wir das einschränken. Die Sicht - auch Luthers und wohl auch die der meisten seiner Zeitgenossen - ist und bleibt mittelalterlich fremd ${ }^{3}$ und voller Vorurteile und Teufelsbilder ausgestattet.

Andererseits: Viele Sprachbilder unserer heutigen Sprache entwickeln sich in dieser Zeit; Bilder, die wir heute nur noch metaphorisch oder als Hyperbel und Klimax verstehen - eine Übertreibung, eine Steigerung dessen, was wir sagen wollen. Glauben wir angesichts der Bilder, die wir historisch von Auschwitz, Hiroshima oder aktueller von Ruanda oder ganz nahe von Aleppo kennen und

1 Luther, Kolumbus und die Folgen. Welt im Wandel 1500-1600. Katalog des Germanischen National-Museums. Hrsg. von Thomas Eser und Stephanie Armer. Nürnberg 2017, S. 6.

2 Vgl. dazu: Andrew Pettegree: Sinn fürs Praktische. Martin Luther spaltete einst die Kirche - als erster Medienstratege der Welt. In: Hundert Jahre Aufbruch in Europa. Das Zeitalter der Reformation. Hrsg. Klaus Brinkbäumer. Hamburg 2017, S. 62 f.

3 Eser/Armer (wie Anm. 1), S. 12. 
sehen, tatsächlich an die Hölle? Oder spielt sich Hölle als eine existentielle Verzweiflung und Angst nur im Inneren unserer Seele ab?

Wie kommt es, dass in unserem und weit über unseren kulturellen Raum hinaus eine Lehre von der Hölle existiert, wir aber nicht wissen, wie wir heute damit angemessen umgehen sollen? Ein Phänomen, das nahezu alle monotheistischen Religionen kennen. Die Geschichte des Himmels und der Hölle und von allem, was dazwischen liegt, ist mehr als ein spezieller theologischer Topos, sie ist vielmehr Teil der allgemeinen Kulturgeschichte.

Die gleichmäßige Ausrichtung über große Teile Europas im 15. Jahrhundert an der Schwelle zur Neuzeit brachte ein übereinstimmendes, von den religiösen Autoritäten konstruiertes Bild des Dies- und des Jenseits. Als Grundlage unserer Vorstellung von Himmel und Hölle dienen dem jüdisch-christlichen Abendland die Bibel und ihre Umfeldschriften.

Der Mensch scheint zunächst - im Paradies - weder unsterblich noch sterblich gewesen zu sein. Da haben wir die Geschichte des Baums der Erkenntnis, der mit einem göttlichen Verbot belegt ist. Durch den Genuss der Frucht kann man letztlich sagen, dass dieser Baum zu einem Baum des Todes wird, denn von nun an sind - in der religiös-mythischen Vorstellung - Menschen sterblich. Der Mensch überlegt und nimmt, trotz Verbots, die Frucht. Der Ursprung der menschlichen Sterblichkeit kann nach dieser Lesart also als selbstverschuldet betrachtet werden. Darüber hinaus ist dieser unerhörte Vorfall erblich und wird von Mensch zu Mensch vererbt. Dem Tod - also der Strafe Gottes - kann niemand entrinnen. Dieser Ursprung der Sterblichkeit hat etwas Negatives. Daraus erklärt sich dann auch, dass das diesseitige Leben nicht das eigentliche Leben sein kann. Das endliche Leben ist nicht die Erfüllung. Nach dem Tod liegt das Ziel.

Daraus ergeben sich einige Konsequenzen, die wir in ihrer ins Phantastische gehende Ausprägung und ausdifferenzierten und überzüchteten Vielfältigkeit bis ins 15. Jahrhundert besonders stark wahrnehmen. Das Jenseits als Folge des sündhaften Lebens hier ist durch die Lehren der Kirche zu einem Ort des Schreckens ohne Trost geworden. Niemand versucht Gründe zu finden, weshalb man vor der anderen Welt nicht zittern braucht; vielmehr wird bis ins hohe Mittelalter ständig daran erinnert, warum man die Strafen im Jenseits fürchten muss.

In der traditionellen Glaubenslehre werden die Vorstellungen von den letzten Dingen in einen zeitlich-systematischen Zusammenhang gestellt. Es gibt ein Hier und Jetzt. Dieses ist mühselig, anstrengend, fehlerhaft, sündig - aber es ist auch endlich: es ist eine Strafe durch Verfehlung im Paradies. Und es gibt ein Dann und Später. Dieses ist gut oder schlecht, Himmel oder Hölle - in jedem Fall aber unendlich: ewig. Dazwischen liegt das Sterben. Der Tod lässt die Grenze überschreiten.

Zur mittelalterlich-neuzeitlichen Wendezeit hat man das unumstößlich scheinende Bild, dass sich im Augenblick des Todes Seele und Leib trennen. Dies ist - ich will es betonen - eine Konstruktion. Die Seele entweicht dem toten Körper. Die Seele tritt vor einen Richter, um dort einem „persönlichen Gericht“ 
vorgeführt zu werden. Maßgeblich für das göttliche Urteil ist in dieser Vorstellung die Lebensbilanz des Verstorbenen (vgl. Altar in der Sakristei in St. Michael). Vor allem der Glaube und das Handeln des Verstorbenen treten unmittelbar hervor.

Die Seelen der schwer Schuldigen sollten in die Verdammnis (Hölle) gestoßen werden. Dort sind sie dann den Qualen und Folterungen der Teufel ausgeliefert; insbesondere aber leiden sie unter der Gottesferne. Sie werden auf ewig gemartert und gefoltert.

Dies kann aber auch abgewendet werden: wer nämlich in vollkommener Güte und im rechten Glauben stirbt, gelangt unmittelbar zur „Anschauung Gottes“. Oft stellt man sich dieses Aufgehobensein ,in Abrahams Schoß“ in Form eines Kindes vor. Die Seele ist schützenswert, verletzlich und wird geschützt und umsorgt. Auch in der polytheistischen Religion der Ägypter spielt das Jenseits eine nicht unerhebliche Rolle. Die Unversehrtheit des Körpers und das Weiterleben im Jenseits ist dort ein wesentliches Merkmal.

Das Diesseits und das Jenseits treten also in eine unmittelbare Kausalität. Wir können hierzu Bilder von Hieronymus Bosch heranziehen (etwa „Der Garten der Lüste").
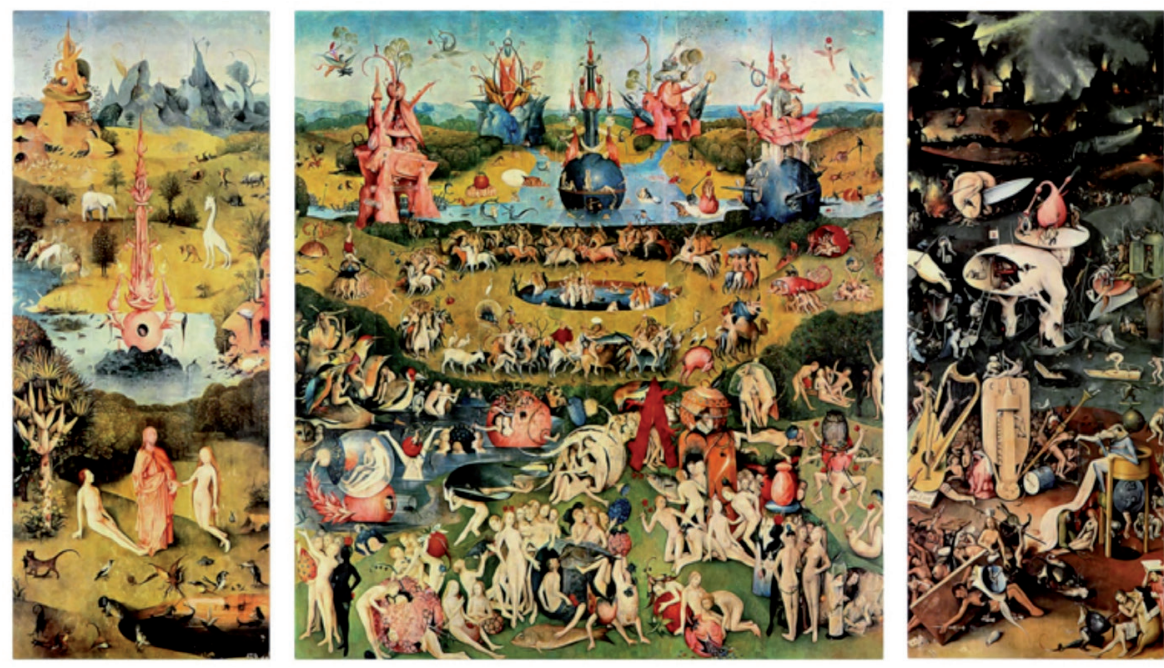

https://upload.wikimedia.org/wikipedia/commons/a/ae/El_jard\%C3\%ADn_ de_las_Delicias\%2C_de_El_Bosco.jpg, abgerufen 12.03.2017.

Aber auch der Strafvollzug im Diesseits ähnelt bereits einem Bild, das man sich vom Jenseits machte.

Die Höllenvorstellung ist alttestamentlich. In der Königszeit hat man hier eine heidnische, gottferne Opferstätte errichtet, an der für den Gott Moloch Kinder 
durchs Feuer gehen mussten (2 Könige 23,10, ${ }^{4}$ Jeremia 32,35 $)$. Die Existenz eines solchen Strafortes steht also fest. Als Gottes Strafe für diesen nicht legitimen Kult soll das Tal zu einem Leichenfeld werden und „Tal des Würgens“ heißen. (Jeremia 7,31 ff.; 19,6). Diese historische Stätte in der Nähe Jerusalems wird in der Apokalyptik zum Bild für die Unterwelt, die Hölle (äth. Henoch 27,2).

Im Neuen Testament finden wir die Traditionen des Alten Testaments fortgeführt: Der jenseitige Straf-Ort für die Sünder wird als „Hölle“, „ewiges Feuer“ (Mt. 18,8; 25,41), „unauslöschliches Feuer“ (Mk. 9,43; Lk. 3,17), „Feuerofen“ (Mt. 13,42) und „Feuersee“ bezeichnet. Diese Darstellung hat im Laufe der Jahrhunderte in den sprachlichen aber vor allem in den künstlerischen Darstellungen eine erhebliche Rolle gespielt.

Aus apokalyptischer Vorstellung ist die Hölle jedoch kein Ort, sondern nimmt eine radikale Krisis des Menschen auf. Der Einzelne kann und muss sein Schicksal selbst beeinflussen. Was diesseits geschieht, ist im Tod unumkehrbar. Dieses zentrale konditionale Verhältnis der Frömmigkeit findet sich in der Erziehung und der Lebenshaltung des Mittelalters wieder. Unbedingter Gehorsam gegen alle religiösen Regeln und Vorschriften, von ihr abgeleitet und abhängig die staatliche Ordnung - bis hin zum Gehorsam gegen Mutter und Vater - sind die Folge. Auch Martin Luther hat diese Auffassung erlernt und verinnerlicht.

Der 1483 geborene Luther beginnt im Sommersemester (April oder Mai 1501) in Erfurt auf Wunsch des Vaters ein Jurastudium, da der Bergmann Hans Luther in seinem Betrieb einen Juristen brauchen kann. Erfurt hat - wie Köln - damals schon eine städtische Universität. Sie ist seit 1392 bereits die fünfte deutsche Uni (nach Prag, Wien, Heidelberg und Köln). Der Eintrag in die Matrikel lautet: Martinus ludher ex mansfeld. Luther schafft sein Grundstudium in der kürzest möglichen Zeit und schließt im September 1502 mit dem Bakkalaureus ab.

Als nächsten Schritt beschließt er Ende 1504 oder Anfang 1505 die Magisterprüfung als zweitbester von siebzehn Kandidaten. Nun beginnt er das eigentliche Jurastudium am 19. Mai 1505 mit dem Zivilrecht. Er durchlebt eine Krise, weil einige seiner Kollegen und Professoren unvermittelt u. a. an der Pest sterben. Er beschäftigt sich theologisch mit der heiligen Anna, die als Schutzpatronin der Bergleute - biographisch naheliegend - der Vater Hans Luder ist Bergmann. Die heilige Anna ist aber auch Schutzheilige gegen einen frühen Tod.

In der mittelalterlichen oder auch frühneuzeitlichen Vorstellung sind früher Tod oder Krankheiten, Missernten, Missbildungen, Unfälle, schwere Geburten, Räuber auf direkte Sünden (z. B. Häresie, falscher Glaube) zurückzuführen und werden weitgehend als göttliche Strafe empfunden. Bei Luther etwa: Am 2. Juli

4 Auch die Opferstätte Tofet im Hinnomtal verwüstete Josia, damit niemand mehr dort seinen Sohn oder seine Tochter für den Götzen Moloch als Opfer verbrennen konnte.

5 Und nicht genug damit: Im Hinnomtal errichteten sie Opferstätten für Baal und verbrannten ihre Kinder als Opfer für den Gott Moloch. Niemals habe ich ihnen so etwas befohlen, mit keinem Gedanken je daran gedacht, dass sie sich in so entsetzliche Schuld verstricken sollten! Damit haben sie ganz Juda zur Sünde verführt. 
1504 gerät er in ein schweres Gewitter und ruft in seiner Angst zur Heiligen Anna, sie möge helfen, und er verspricht Mönch zu werden. Er tritt am 17. Juli 1505 in das Erfurter Kloster der Augustiner-Eremiten ein.

Besonders zu betonen ist dabei, dass sich Luther - und das steht ebenfalls für das vorreformatorische Jenseitsbild - nicht direkt an Gott wendet. Er wendet sich an Anna, die Mutter Marias, die Großmutter Jesu. Die Vorstellung, dass es eines Fürsprechers auf der Himmelstreppe bedarf, ist hier das zentrale Moment. Für sein Anliegen sind Zwischenschritte nötig: Anna nimmt auf, wendet sich mit dem Anliegen an Maria, diese an Jesus. Luther verspricht sich Jesus! Ein Dreischritt! In vielen Gemälden finden wir diese Fürsprache-Überlegung. Ich opfere mich, wie Maria sich als Mutter zur Verfügung stellt, wie Jesus sich opfert (Kreuzestod). Auch am Jüngsten Tag braucht man einen Fürsprecher - dazu aber später.

Luthers Schritt, nach einer vom Vater vorgesehenen Juristenausbildung, die sehr schnell, sehr erfolgreich und schon sehr weit fortgeschritten war, sein Schritt also dem Vater den Gehorsam zu verweigern und Mönch zu werden, ist in diesem Zusammenhang als außergewöhnlich, geradezu unerhört zu sehen.

Der von außen kommende, als Gotteszeichen verstandene Blitz, ist das Entscheidungszeichen für ihn. Nun steht er in einem schier unüberwindlichen Spannungsfeld: entweder dem Vater den Gehorsam zu verweigern oder sein Versprechen gegenüber der Heiligen Anna, zu der er gebetet hat, zu brechen. Das gegenüber dem Himmel gegebene Versprechen wiegt - auch im Hinblick auf die Erziehung und die Jenseitsvorstellung - mehr. Hier ist Luther ganz in der mittelalterlichen Frömmigkeit verhaftet. Die ganze Lebenssituation hat dadurch etwas durchaus antik Dramatisches.

Luther wird Mönch. Er ist tiefgläubig. Die Lehren gelten ihm unmittelbar. Er gerät auch als Mönch in Gewissenskonflikte, weil bei strenger mönchischer Lebensweise nicht alle Stundengebete und Dienste abzuleisten sind. Er merkt, dass er es nicht vermag, alle Regeln zu befolgen und muss - in seiner bekannten Dies-Jenseits-Vorstellung - befürchten, zu scheitern und im Jenseits bestraft zu werden. Das Diesseits beeinflusst das Jenseits. Um im Jenseits bewahrt zu sein, ist ein permanentes Beten für das Seelenheil angebracht. Fürsten und Adelige richten Stiftungen ein - ewiges Beten für die Seele - . Klöster und Stifte werden gegründet und mit Pfründen ausgestattet.

\section{Himmel und Hölle}

Der Tod ist die Trennung des Menschen in einen Leib und eine Seele. Der Leib wird begraben und am Jüngsten Tage wird entschieden (Mt. 13, 49, 50). ${ }^{6}$ Jeder 
werde bekommen, was er im Leben verdient hat (2. Kor. 5, 107). Die Trennung bleibt nicht bestehen. Die Gräber werden sich auftun und die Leiber werden sich mit den Seelen zu einem neuen Leib vereinen. Aus diesem Grund werden im Mittelalter auch Beinhäuser angelegt, um den Weg ins Paradies am Jüngsten Tag, wenn sich die Gräber öffnen, nicht zu gefährden. Seele und Leib werden zu einem neuen Leib. Wenn aber der Leib nicht mehr da ist, kann kein neuer Leib entstehen, keine Auferstehung erfolgen. Dieser Dualismus von Leib und Seele hat bereits im Diesseits eine hohe Bedeutung. Glaubt die Seele nicht ganz und gar, muss der Leib bestraft werden (Krankheit, früher Tod etc.). Der Körper ist also eine Art Ausdrucksmedium der Seele.

Nach dem Tod findet die Seele zunächst zu einem ersten persönlichen Gericht. Hier wird das im Leben geschaffene gewogen. Eine Vorentscheidung wird getroffen:

Die Seele kommt in den Himmel (in Gemeinschaft mit Gott); die Seele kommt in die Hölle (wo Gott nicht ist); die Seele kommt in eine Zwischenstation zur Reinigung.

Diese Vorstellung basiert auf Mt. 3, 11. Als Johannes der Täufer sagt: „Ich taufe euch nur mit Wasser (zum Zeichen) der Umkehr. Der aber, der nach mir kommt, ist stärker als ich und ich bin es nicht wert, ihm die Schuhe auszuziehen. Er wird euch mit dem Heiligen Geist und mit Feuer taufen.“

Während Himmel und Hölle biblisch belegt sind, kann das Fegefeuer nicht direkt aus der Heiligen Schrift entnommen werden. Die eben zitierte Stelle aus Mt. 3,11 kann als reinigende Buße verstanden werden. Dieser zeitlich begrenzte Klärungs- und Läuterungsort - das (Fege)Feuer - behält weitgehend einen Strafcharakter, insofern, als die Ableistung der Bußauflage der Vergebung der Sünden dienen soll. Es muss wehtun, wenn es helfen soll.

Aber: Die Buße oder Strafe kann verkürzt oder aufgehoben werden. Mit dem rein auf ökonomische Hascherei ausgelegten Ablassbrief wird im Laufe des frühen 16. Jahrhunderts ein neuer Höhepunkt erreicht.

Die Lehre vom Fegefeuer knüpft biblisch an 1 Kor 3,13-15 an, wo die Werke des Einzelnen beim Gericht im Feuer geprüft werden. Im 12. Jahrhundert ist die Vorstellung eines Fegefeuers (von „fegen“ - entfernen; also entfernen der Sünden; reinigen) endgültig im Volksglauben verankert.

7 Denn einmal werden wir uns alle vor Christus als unserem Richter verantworten müssen. Dann wird jeder das bekommen, was er für sein Tun auf dieser Erde verdient hat, mag es gut oder schlecht gewesen sein.

8 Das Werk eines jeden wird offenbar werden; jener Tag wird es sichtbar machen, weil es im Feuer offenbart wird. Das Feuer wird prüfen, was das Werk eines jeden taugt. ${ }^{14}$ Hält das stand, was er aufgebaut hat, so empfängt er Lohn. ${ }^{15}$ Brennt es nieder, dann muss er den Verlust tragen. Er selbst aber wird gerettet werden, doch so wie durch Feuer hindurch. 
Hier verstehen wir nun auch die Strafe des Hexenverbrennens im Mittelalter. Während der sündige Körper - eigentlich ,gereinigt" wird -, wird er in Wirklichkeit verbrannt und vernichtet, da er ohnehin nicht in den Himmel eingehen wird. Der Hexenleib braucht deshalb auch nicht bewahrt werden. So erfährt die Seele durch das Feuer eine Reinigung und gleichzeitig eine Strafe bzw. einen Vorgeschmack der Hölle. Ein diesseitiges Verbrennen hat so die Wirkung, dass die Auferstehung - nach vorreformatorischer Vorstellung - verhindert wird. Diese Vorstellung finden wir in 1 Joh 3,14-15: , ${ }^{14}$ Wir wissen, dass wir aus dem Tod in das Leben hinübergegangen sind, weil wir die Brüder lieben. Wer nicht liebt, bleibt im Tod. ${ }^{15}$ Jeder, der seinen Bruder hasst, ist ein Mörder und ihr wisst: Kein Mörder hat ewiges Leben, das in ihm bleibt.“

Gott macht darauf aufmerksam, dass Sünder (der Erbsünde im Paradies wegen also alle Menschen) von ihm getrennt werden, wenn sie es unterlassen, sich der schweren Nöte der Armen und Geringen, die ihrer Brüder und Schwestern anzunehmen (vgl. ,Vom Weltgericht“"Mt 25,31-46).

Mt. 25, 31ff.: Weltgericht am Jüngsten Tag; alle Menschen erstehen vom Tod auf; nach den Taten des Lebens richtet Jesus; Gute in den Himmel, Böse in die Hölle

\section{Vom Weltgericht Mt. 25}

${ }^{31}$ Wenn der Menschensohn in seiner Herrlichkeit kommt und alle Engel mit ihm, dann wird er sich auf den Thron seiner Herrlichkeit setzen. ${ }^{32}$ Und alle Völker werden vor ihm zusammengerufen werden und er wird sie voneinander scheiden, wie der Hirt die Schafe von den Böcken scheidet. $2^{33}$ Er wird die Schafe zu seiner Rechten versammeln, die Böcke aber zur Linken. ${ }^{34}$ Dann wird der König denen auf der rechten Seite sagen: Kommt her, die ihr von meinem Vater gesegnet seid, nehmt das Reich in Besitz, das seit der Erschaffung der Welt für euch bestimmt ist. ${ }^{35}$ Denn ich war hungrig und ihr habt mir zu essen gegeben; ich war durstig und ihr habt mir zu trinken gegeben; ich war fremd und obdachlos und ihr habt mich aufgenommen; ${ }^{36}$ ich war nackt und ihr habt mir Kleidung gegeben; ich war krank und ihr habt mich besucht; ich war im Gefängnis und ihr seid zu mir gekommen. ${ }^{37}$ Dann werden ihm die Gerechten antworten: Herr, wann haben wir dich hungrig gesehen und dir zu essen gegeben, oder durstig und dir zu trinken gegeben? $3^{38}$ Und wann haben wir dich fremd und obdachlos gesehen und aufgenommen, oder nackt und dir Kleidung gegeben? ${ }^{39}$ Und wann haben wir dich krank oder im Gefängnis gesehen und sind zu dir gekommen? ${ }^{40}$ Darauf wird der König ihnen antworten: Amen, ich sage euch: Was ihr für einen meiner geringsten Brüder getan habt, das habt ihr mir getan. ${ }^{41}$ Dann wird er sich auch an die auf der linken Seite wenden und zu ihnen sagen: Weg von mir, ihr Verfluchten, in das ewige Feuer, das für den Teufel und seine Engel bestimmt ist! ${ }^{42}$ Denn ich war hungrig und ihr habt mir nichts zu essen gegeben; ich war durstig und ihr habt mir nichts zu trinken gegeben; ${ }^{43}$ ich war fremd und 
obdachlos und ihr habt mich nicht aufgenommen; ich war nackt und ihr habt mir keine Kleidung gegeben; ich war krank und im Gefängnis und ihr habt mich nicht besucht. ${ }^{44}$ Dann werden auch sie antworten: Herr, wann haben wir dich hungrig oder durstig oder obdachlos oder nackt oder krank oder im Gefängnis gesehen und haben dir nicht geholfen? ${ }^{45}$ Darauf wird er ihnen antworten: Amen, ich sage euch: Was ihr für einen dieser Geringsten nicht getan habt, das habt ihr auch mir nicht getan. ${ }^{46}$ Und sie werden weggehen und die ewige Strafe erhalten, die Gerechten aber das ewige Leben. ${ }^{9}$

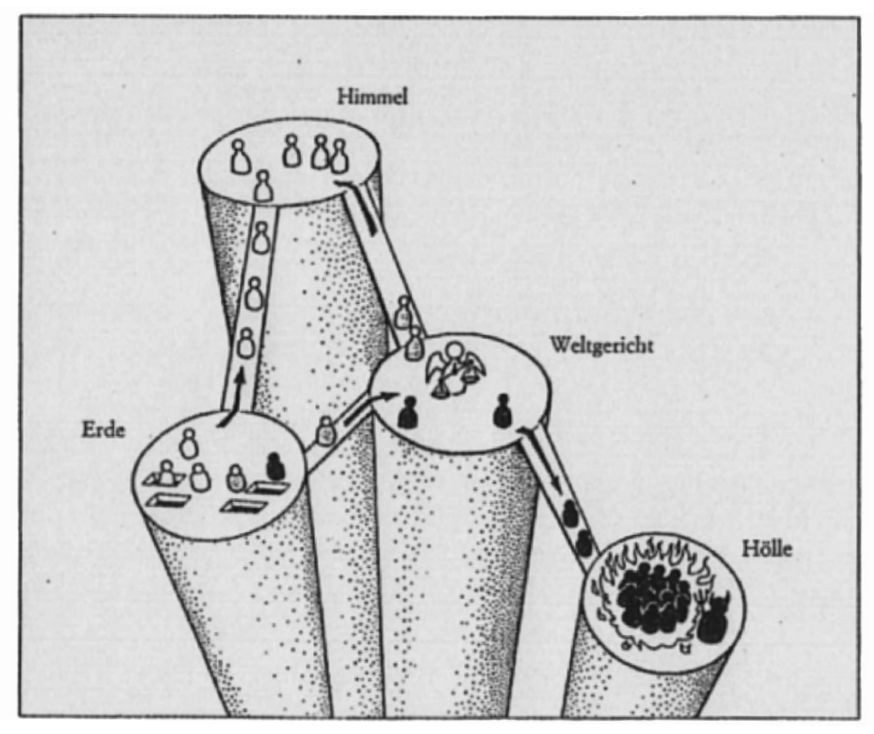

Joh. 5, 24-29: Johannes kennt neben Guten und Bösen auch Halbgute; am Jüngsten Tag müssen nur die Bösen und die Halbguten vors Weltgericht. Die Guten kommen sofort in den Himmel, die Bösen und Halbguten werden - je nach Taten - gerichtet (Himmel oder Hölle)

Mit der Festigung der Fegefeuerlehre wird die Jenseitstopographie komplexer: Zunächst soll es nun ein individuelles Partikulargericht geben (Heilige direkt in den Himmel, Böse direkt in die Hölle). Neu ist jetzt: Arme Seelen kommen zur Läuterung in das Fegefeuer und sind - nach angemessener Buße - hoffnungsfroh zur Auferstehung; ungetaufte Kinder kommen in den Limbus (neutraler Ort).

Die Fegefeuerlehre wurde dann mit der Weltgerichtvorstellung verbunden. So entstand „Gegenverkehr“ auf dem Weg in den Himmel und in die Hölle.

9 Peter Jezler: Himmel, Hölle, Fegefeuer. Das Jenseits im Mittelalter (Ausstellungskatalog), Zürich 1994. 


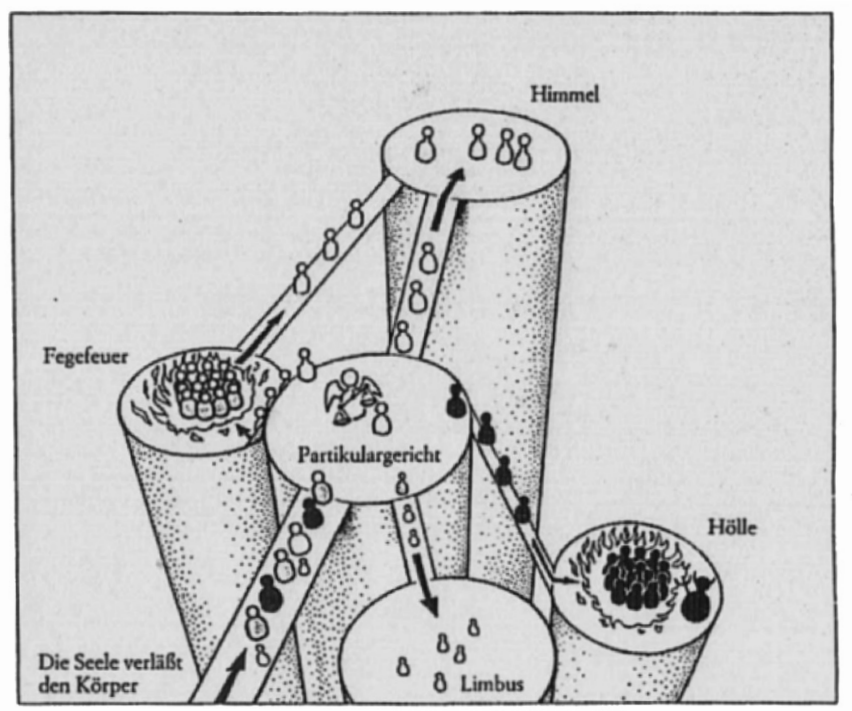

Durch das Partikulargericht kommen alle Seelen entweder direkt in die Hölle oder ins Fegefeuer. Ausnahmen (Heilige). Am Jüngsten Tag (Weltgericht) wird dann für alle Seelen abschließend entschieden!

In Todsünde sterben, ohne seine Sünden bereut zu haben und ohne die barmherzige Liebe Gottes anzunehmen, bedeutet im Mittelalter: durch eigenen freien Entschluss für immer von Gott getrennt bleiben. Diesen Zustand der endgültigen Selbstausschließung aus der Gemeinschaft mit Gott und den Seligen durch eigenes Fehlverhalten nennt man „Hölle“. Aus diesem Grund sind auch Feuerbestattungen bis heute nach katholischer Ansicht kontraproduktiv.

Schließlich wird am Jüngsten Tag, am Ende aller Zeiten ein Weltgericht abgehalten; hier entscheidet Jesus als Richter, wer abschließend in den Himmel bzw. in die Hölle kommt. Auf das apokryphe Nikodemus-Evangelium schließlich geht die Vorstellung von Michael als Hüter des Paradiestores zurück.

Auch wird Michael mit dem ,Engel des Angesichts“ in Verbindung gebracht, der nach der Apokryphe der Jubiläen zunächst von Gott als Chronist der Weltgeschichte eingesetzt worden ist (Jub 1,27), dann aber wiederum auf Gottes Geheiß Mose beauftragt hat, die Schöpfungsgeschichte für die Menschen niederzuschreiben (Jub 2,1).

In den 1947 entdeckten Schriftrollen vom Toten Meer wird Michael als „Fürst des Lichts“ bezeichnet, der die Heerscharen Gottes gegen die Mächte des Bösen unter Belial führt. Auch trägt er dort den Titel ,Vizekönig des Himmels“.

Daneben spielt Michael eine wichtige Rolle im Volksglauben. Er ist es, der ein Verzeichnis der guten und schlechten Taten eines jeden Menschen erstellt, das diesem zunächst am Tag des Sterbens (Partikulargericht), aber auch am Tage des Jüngsten Gerichts vorgelegt wird und auf dessen Basis er über ihn richtet. Er 
erscheint hier in der wichtigen Position des Seelenwägers. Auch geleitet er die Seele des Verstorbenen auf ihrem Weg ins Jenseits. Dementsprechend wird er mit den Attributen Waage und Flammenschwert dargestellt. Die darstellende Kunst ordnet ihm die Farbe Rot in allen Schattierungen zu (für Feuer, Wärme und Blut). An dieser Stelle sei mir ein kleiner Einschub erlaubt, da wir uns ja in einer Freien Reichsstadt mit einer Michaelskirche befinden. Als Otto I. im Kampf gegen die Ungarn auf dem Lechfeld eine Niederlage drohte, betete er zu Gott und bat die Erzengel um Hilfe. Otto gewann die Schlacht und bestimmte den Erzengel Michael zum Schutzheiligen des Reiches. Aus mittelalterlicher Sicht ganz schlüssig und mit legitimem Hintergedanken. Wenn es am Jüngsten Tag zur Verhandlung und zum Abwiegen der diesseitigen Verdienste kommt, schadet es nicht, wenn man mit dem Waaghalter bereits eng verbunden ist und einen Fürsprecher hat.

Michael soll Anwalt sein, der Jesus am Jüngsten Gericht ganz nahe sein wird. Michael - Jesus - Gott. Da haben wir ihn wieder, den Dreischritt.

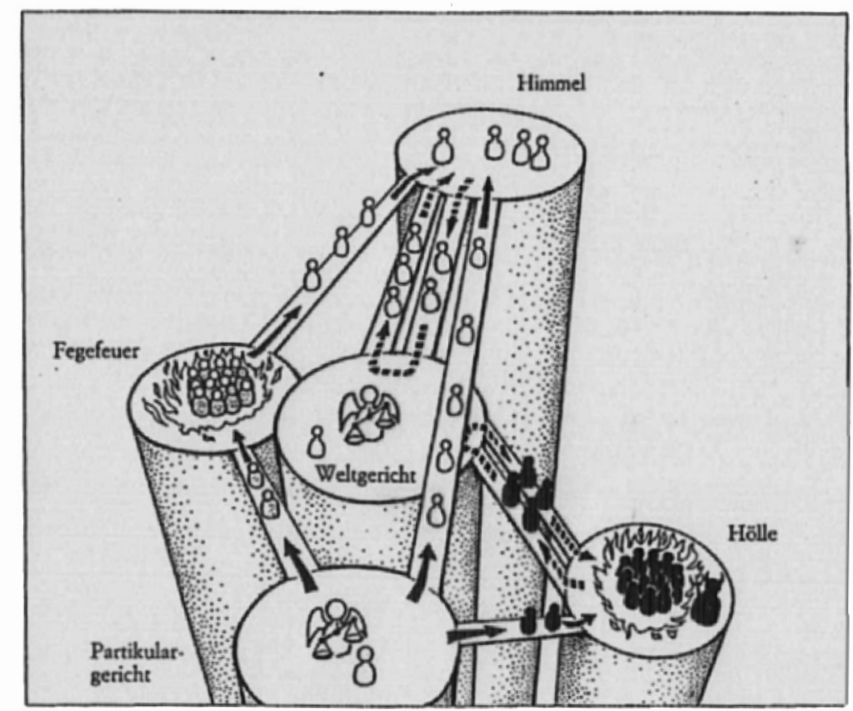

Die Freien Reichsstädte waren im Mittelalter direkt dem römischen Kaiser unterstellt und fühlten sich demnach auch diesem Schutzpatron besonders verbunden. In nahezu allen Freien Reichsstädten ist eine Michaelskirche die Hauptkirche. Das ist nachvollziehbar.

Zurück zum letzten Gericht. Bei diesem Weltgericht - dem „Jüngsten Tag“ - führt Gott die Menschheit zu einer großen Gemeinschaft zusammen. Gott stellt endgültig die Herrlichkeit der Erlösten (den „Neuen Himmel“) her und verstößt endgültig die Verdammten. Die gesamte Schöpfung wird umgewandelt in ein vollkommenes Werk Gottes. Bei der Auferstehung am Ende der Zeiten erhalten alle Menschen wieder einen Körper. Ob dazu die alten Knochen gebraucht werden 
oder nicht, konnte man sich nicht genau vorstellen. Aber zur Sicherheit - um im Jenseits bestehen zu können - wurden die Gebeine in Gebein-Häusern aufbewahrt.

Die Lebenszeit Luthers fällt zusammen mit der Zeit der Renaissance, der Rückbesinnung auf antike Vorstellungen, der Wiedergeburt des antiken Wissens. Die Ambivalenz zwischen tiefer, bewahrender Frömmigkeit und rückwärtsblickendem Vorwärtsstreben macht sich auch an der Lebensunsicherheit des 15. und 16. Jahrhunderts aus. Tod, Krankheit, Pest, Kriege als Anlässe zur Verzweiflung, Verunsicherung, Demut, aber eben auch zu Mut, Entdeckerlust und Betrachtung der Schönheit der Welt führen zu einer radikalen Einstellung gegenüber dem Tod. Verstorbene leben nach dem Tod weiter; Tod als Unsterblichkeit, Tod als Auslöschung der Menschheit - die Apokalypse ist nahe.

Die Dekaden vor Beginn der Reformation, also die Jahre um 1500, können als eine Epoche der verstärkten Höllenfurcht angesehen werden. Naturkatastrophen und Pestepidemien, Missernten, Hungersnöte und damit verbunden das Gefühl, in einer apokalyptischen Endzeit zu leben, führten zu intensiven Lebens- und Todesängsten. Viele empfanden den Teufel allgegenwärtig und lebten entsprechend in der Furcht vor ewigen Höllenstrafen.

Von Seiten der Kirche wird dem nicht etwa mit seelsorgerlichem Trost entgegengesteuert, sondern die Angst der Menschen zur Stärkung der eigenen kirchlichen Machtpositionen - aus heutiger Sicht - missbraucht. Eine tiefe Kluft zwischen frommen Gläubigen und säkularen Kirchenführern bricht auf. Vor allem die aus Machtkalkül installierten Päpste machen den Unterschied deutlich. In Rom herrscht seit 1503 Papst Julius II., der mit aller Kraft die weltliche Macht und das Territorium des Kirchenstaates ausbauen will. Er lässt auch den ca. 1200 Jahre alten Petersdom abreißen, um eine neue Kirche zu errichten.

Die Reformation in Deutschland wird heute datiert auf den 31. Oktober 1517 mit Luthers 95 Thesen, deren Ausgangspunkt der Streit um den päpstlichen Ablasshandel ist. Mit dem Erwerb sogenannter Ablassbriefe können die Gläubigen sich von Fegefeuerstrafen freikaufen oder diese zumindest verkürzen. Viele Menschen glauben das aus der bisherigen Jenseitsvorstellung heraus. Sie sind sogar davon überzeugt, dass sie bereits Verstorbene vor Höllenstrafen retten können. Als Kind seiner Zeit glaubt auch Luther fest an eine Hölle als dem Ort der Gottesferne und der ewigen Qualen für die Ungläubigen.

Die direkte Konfrontation des ,,verkommenen Papstes“ und der liederlichen und sehr weltlichen Zustände des Klerus in Rom und der eifrige Handelsbetrieb mit Ablässen, den er bei seiner Reise im Jahr 1510/11 nach Rom kennenlernt, lässt ihn zweifeln - ja fast verzweifeln. Nicht am Glauben, sondern an den Repräsentanten der Kirche. Er soll Streitigkeiten des Klosters klären lassen. Aber er sieht eine verführte, vom Teufel besessene Kirchenzentrale in der ewigen Stadt. So, darin war sich Luther gewiss, werde die Seele nicht gerettet werden können. 
Erasmus von Rotterdam wird das in seinem Werk von 1520 „Papst Julius vor der Himmelstür"10 überzeichnen:

Julius: Was, zum Teufel, ist denn hier los? Wieso geht die Tür nicht auf? Irgendwer muss das Schloss ausgetauscht oder kaputtgemacht haben! [...]

Petrus: Ein Glück, dass wir eine Pforte hart wie Diamant haben. Sonst hätte uns so ein Kerl, wer immer es ist, jetzt die Tür eingetreten. Es muss wohl [...] ein Städtezertrümmerer sein. Aber, großer Gott, was für ein Kloakengestank steigt mir in die Nase! [...] Wer bist du? Und was willst du?

- Der Disput geht noch eine Weile weiter. Schließlich:

Julius: Schluss mit dem Geschwätz, sage ich! Wenn du nicht sofort parierst, werde ich den Blitzstrahl der Exkommunikation auf dich - ja auf dich! schleudern, mit dem ich einstmals die größten Könige und sogar ganze Reiche in Furcht und Schrecken versetzt habe. Siehst du hier die Bulle, die bereits zu diesem Zwecke bereit steht?

Petrus: Von welchem fürchterlichen Blitz und Donner redest $d u d a$, von welchen Bullen, und warum so geschwollen? Von diesen Dingen hat uns Christus nichts erzählt [...].

Petrus: [...] Doch, sag einmal, du drohst mir mit dem Blitzstrahl der Exkommunikation? Mit welchem Recht, wenn ich fragen darf?

Julius: Mit dem besten von der Welt, da du ja nicht mehr im Amt bist und nicht mehr als ein einfacher Priester [...] weil du nicht einmal die Messe feiern kannst.

Petrus: Wohl deshalb, weil ich nicht mehr unter den Lebenden bin.

Julius: Aber sicher!

Petrus: Aber aus genau demselben Grund hast du mir doch ebenso wenig voraus wie irgendein anderer Toter.

Noch ist es aber bei Luther nicht soweit: Aus Furcht vor Teufelsbeeinflussung, und im festen Glauben, dass die Kirche in Händen von Verführern sei, wendet er sich ab. Wer sich diesem Sündenpfuhl anschließt, muss drüben verloren sein. Mit dieser Ansicht ist Luther ganz und gar auf Linie der Lehrmeinung der Kirche! Dabei ist nicht an Bruch oder Revolte gedacht. Es geht Luther zunächst um

10 Erasmus von Rotterdam: Papst Julius vor der Himmelstür. Julius exclusus e Coelis. Lateinisch und deutsch übersetzt, mit Anmerkungen und einem Nachwort von Werner von Koppenfels. Mainz 2011, S. 7 f. 
Rückführung auf den biblischen Glauben, ${ }^{11}$ den wahren und echten Glauben, der am Ende zum Seelenheil führt. Wie einst in der Auseinandersetzung, wem er folgen soll - seinem Vater oder seinem der Heiligen Anna gegebenen Versprechen -, so entscheidet er sich auch jetzt für die höhere Autorität: die Bibel.

Besonders einschneidend ist in diesem Zusammenhang die Auseinandersetzung mit dem Römerbrief. Im Frühjahr 1513 findet er beim Bibelstudium im Römerbrief die Sätze, die ab sofort sein Denken beeinflussen. Die erste Stelle findet er im Römerbrief 3, 21-24:

${ }^{21} N u n$ aber ist ohne Zutun des Gesetzes die Gerechtigkeit, die vor Gott gilt, offenbart, bezeugt durch das Gesetz und die Propheten. ${ }^{22}$ Ich rede aber von der Gerechtigkeit vor Gott, die da kommt durch den Glauben an Jesus Christus zu allen, die glauben. Denn es ist hier kein Unterschied: ${ }^{23}$ sie sind allesamt Sünder und ermangeln des Ruhmes, den sie bei Gott haben sollten, ${ }^{24}$ und werden ohne Verdienst gerecht aus seiner Gnade durch die Erlösung, die durch Christus Jesus geschehen ist.

Die Schlüsselstelle für Luther steht in Römer, 1,17:

${ }^{1}$ Denn darin wird offenbart die Gerechtigkeit, die vor Gott gilt, welche kommt aus Glauben in Glauben; wie geschrieben steht (Habakuk 2,4): „Der Gerechte wird aus Glauben leben."

Die 1521 auf der Wartburg angefertigte Übersetzung des Neuen Testaments und 1523 die des Alten Testaments hatten eine in sich schlüssige Formel. Um im Verstehen zum Seelenheil zu gelangen, dort gnädig aufgenommen zu werden, müsse jeder selbst lesen und verstehen, was in der Bibel steht. Dazu bedürfe es einerseits keiner Fürsprecher oder Mittler und andererseits nur der Heiligen Schrift. Also müsse man Menschen zum Lesen und Verstehen befähigen. So ist es nur folgerichtig, dass Schulen und Bildung zum zentralen Volksbildungsansatz der Reformation wurden. Dazu hören wir aber an anderer Stelle Kompetentes. Ausgangspunkt bleibt Gott der Schöpfer und seine Gnade. Luther lenkt den Fokus auf „Jesus Christus allein“. ${ }^{12}$ Neu ist nun die Ansicht, dass der Himmel allen gemeinsam ist und für alle derselbe.

Allein durch seinen Glauben an Christus ist der Mensch gerettet, weil die Rechtschaffenheit und der Opfertod Christi auf alle übertragen worden sei. Wenn man stirbt, dann wird man aus Gnade direkt in den Himmel gehen, um in der Gegenwart seines Herrn zu sein. Dieses neue Bild geht davon aus, dass der Himmel keine Kopie unserer gegenwärtigen Welt sein kann. Die Vorstellung der Trennung von Seele und Körper spielt keine entscheidende Rolle mehr. Gott allein ist Erlöser. Ein Fegefeuer wird nun überflüssig. Und dennoch blieben einige Vor-

11 Eser/Armer (wie Anm. 1), S. 12.

12 Vgl. dazu auch: Martin Jung: Jesus Christus allein. In: Sonntagsblatt. Sonderausgabe des Evangelischen Gemeindeblattes für Württemberg. Hg. von Roland Gertz. Stuttgart 2017, S. 4-12. 
stellungen zunächst erhalten: Bis zum Jüngsten Gericht werden sich die Seelen in einer Art Schlaf befinden.

Nachdem die Reformation auch aus allzu weltlichen Gesichtspunkten durch diverse Fürsten - quasi von oben - ins Laufen kommt, lehnen nahezu alle Reformatoren das Fegefeuer als nicht biblisch ab. Und auch die Hölle soll, so will es Luther, kein herausgehobenes Thema mehr für Predigten sein. Dort soll vielmehr der gnädige Christus als der Retter und Erlöser als positive Lichtgestalt zur Sprache kommen, nicht aber der Teufel in seiner negativen, destruktiven, dunklen Erscheinung.

Es muss gesagt werden, dass Luther die Existenz des Teufels (und damit der Hölle und der Verführer) oder von Hexen nicht verneint. Zahlreiche Satire- und Verunglimpfungsblätter der Reformation und Gegenreformation sind uns bis heute erhalten. Der Propagandakampf bis aufs Messer war todernst. Die propagandistische Darstellung, die uns heute überzeichnet erscheint, darf nicht darüber hinwegtäuschen, dass es im Kern immer noch um den rechten Glauben, den rechten Weg im Diesseits - und damit auch um die ewige Seinsfrage im Jenseits - geht: Himmel oder Hölle.

In dieser Hinsicht wendet sich die Reformation scharf gegen die ausschließliche mittelalterliche Angstreligion. An den Jenseitsvorstellungen bricht die Kirche auseinander. Aber schon die übernächste Generation lutherischer Theologen geht in eine andere Richtung und entfernt sich von Luthers seelsorgerlichem Ansatz und propagierte erneut eine Art Fegefeuer, den sogenannten „Ort der Qualen“, welchem nach dem Gericht die vollständige, ewige Verdammnis folgt. Man kreiert zwar keine neuen Höllenbilder, hält aber daran fest, dass es innerhalb der ewigen Verdammnis graduelle Unterschiede bei den Strafen geben wird.

Die Teufels- und Dämonenvorstellungen werden am Ende des 16. Jahrhunderts wieder intensiviert und führen dazu, dass auch in protestantischen Gebieten Hexen weiter verfolgt und hingerichtet werden. Von Höllenqualen, Höllenrachen und Höllenpein ist sogar noch in vielen Kirchenkantaten Johann Sebastian Bachs und in anderen Kirchenliedern des Barock (u. a. Paul Gerhardt) die Rede, die die Hölle im Bewusstsein vieler evangelischer Christen sehr lebendig werden lassen. Dies hat immer auch mit den Lebensumständen - etwa den Grausamkeiten des 30jährigen Krieges oder auch mit den erneuten Pestepidemien des Barock zu tun. Das Carpe diem und das Memento Mori schaffen eine neue Frömmigkeit. Aber das ist eine andere Geschichte. 